\title{
Abnormal interhemispheric functional connectivity in patients with primary dysmenorrhea: a resting-state functional MRI study
}

\author{
Yanan Zhang ${ }^{1 \#}$, Yiran Huang ${ }^{2 \#}$, Ni Liu ${ }^{1}$, Zhenjia Wang ${ }^{1}$, Junchen $\mathrm{Wu}^{1}$, Wenxun $\mathrm{Li}^{2}$, Jing $\mathrm{Xia}^{2}$, \\ Zhidan $\mathrm{Liu}^{2}$, Yingqiu $\mathrm{Li}^{2}$, Ying $\mathrm{Hao}^{3 *}$, Jianwei Huo ${ }^{1 * \wedge}$ \\ ${ }^{1}$ Department of Radiology, Beijing Hospital of Traditional Chinese Medicine, Capital Medical University, Beijing, China; ${ }^{2}$ School of Acupuncture \\ Moxibustion \& Tuina, Beijing University of Chinese Medicine, Beijing, China; ${ }^{3}$ Beijing International Center for Mathematical Research, Peking \\ University, Beijing, China.
}

Contributions: (I) Conception and design: Y Zhang, Y Huang, Y Hao, J Huo; (II) Administrative support: Y Hao, J Huo; (III) Provision of study materials or patients: Y Huang, Y Hao, W Li; (IV) Collection and assembly of data: Y Zhang, N Liu, Z Wang, J Wu, W Li, J Xia, Z Liu, Y Li; (V) Data analysis and interpretation: Y Zhang, Y Huang, N Liu; (VI) Manuscript writing: All authors; (VII) Final approval of manuscript: All authors.

\#These authors contributed equally to this work.

*These authors contributed equally to this work.

Correspondence to: Jianwei Huo. Department of Radiology, Beijing Hospital of Traditional Chinese Medicine, Capital Medical University, 23 Meishuguanhou Street, Dongcheng District, Beijing 100010, China. Email: huojianwei@bjzhongyi.com; Ying Hao. Beijing International Center for Mathematical Research, Peking University, No. 5 Yiheyuan Road, Haidian District, Beijing 100871, China. Email: Helenh1234@163.com.

Background: Neuroimaging studies have confirmed that functional connectivity (FC) disruption of painrelated brain networks may contribute to the cerebral pathophysiology of primary dysmenorrhea (PDM). However, it remains unclear whether FC of symmetrical regions of bilateral hemispheres associated with PDM is abnormal. This functional MRI study aimed to explore the changes of voxel-mirrored homotopic connectivity (VMHC) and seed-based FC in patients with PDM.

Methods: A cohort comprising patients with PDM ( $\mathrm{n}=35)$ and healthy controls (HCs) ( $=41)$ underwent resting-state functional MRI scans during their menstrual phase. Interhemispheric FC was compared between the two groups using VMHC analysis. Brain areas with significant group differences in VMHC were selected as seed regions for FC analysis. Correlation analysis was also conducted to examine the relationship between abnormal connectivity of brain regions and clinical measures of pain and anxiety.

Results: Compared with healthy individuals, patients with PDM showed significantly enhanced VMHC in the bilateral orbital part of the superior frontal gyrus and the bilateral middle frontal gyrus. Subsequent seedbased FC analysis showed enhanced connectivity between the aforementioned areas and pain-related brain structures. Hyperconnectivity between the left middle frontal gyrus and the right cingulate gyrus in patients was negatively correlated with an increase in the visual analogue score (VAS) for pain $(r=-0.341, \mathrm{P}<0.05)$.

Conclusions: Our findings indicate that ongoing dysmenorrhea is accompanied by abnormal interhemispheric functional coordination and enhanced connectivity in pain-related regions, attention networks, and the reward system. These findings may provide a novel perspective on the central mechanism of pain caused by PDM.

Keywords: Primary dysmenorrhea (PDM); functional magnetic resonance imaging; voxel-mirrored homotopic connectivity (VMHC); functional connectivity (FC)

^ ORCID: 0000-0001-6173-2318. 
Submitted Jul 15, 2021. Accepted for publication Nov 23, 2021.

doi: 10.21037/qims-21-731

View this article at: https://dx.doi.org/10.21037/qims-21-731

\section{Introduction}

Primary dysmenorrhea (PDM) is a general gynecological disorder characterized by cramping pain in the lower abdomen that occurs during menstruation in the absence of other identified pathological conditions such as endometriosis (1). Pain intensity peaks at 24 to 36 hours after the menstrual phase begins and may persist for up to a few days (2). Associated symptoms include nausea and vomiting, fatigue, headaches, dizziness, cold limbs, and syncope and severe pain. Similar symptoms can recur across subsequent menstrual cycles. A well-known pathophysiological process of PDM is an increase in prostaglandins that causes uterine contractions, which restrict blood flow and lead to cramping (2). The prevalence of PDM varies between different countries and regions, and at least one-third of females report moderate or severe menstrual pain, which impacts on their quality of life and productivity in study or work (3-6). Long-term recurrent pain episodes can affect the central nervous system and cause further abnormal changes in brain structures and functional connectivity (FC) (7-9), which may aggravate the dysmenorrhea, forming a vicious cycle. Hence, it is necessary to study the central mechanism of PDM, as this would play a guiding role in future clinical practice.

An increasing number of neuroimaging studies show that abnormal FC of pain-related brain regions and networks may be involved in the central mechanism that underlies PDM (10-14). Recent functional MRI (fMRI) studies discovered alterations associated with PDM in several brain regions including the precuneus, left dorsomedial prefrontal cortex, anterior cingulate cortex, thalamus, and amygdala. Abnormal networks appear to be mainly located in the default mode network, reward system dorsal, and attention networks. Existing findings are mostly focused on the FC between seed regions of interest with all brain voxels. However, FC changes in symmetrical regions of the left and right hemispheres have not been fully investigated.

In 2010, Zuo et al. (15) proposed the voxel-mirrored homotopic connectivity (VMHC) method to measure resting-state FC strength between the mirrored counterpart areas of the bilateral hemispheres, which reflects interhemispheric functional communication and coordination. One study has confirmed that there is a strong correlation between activity in symmetrical brain regions (16). This novel method of resting-state fMRI (rsfMRI) may yield new insights about both disease processes and healthy development. VMHC impairment has been reported in pain-related diseases, such as postherpetic neuralgia (17), acute eye pain (18), and chronic lower back-related leg pain (19). However, little is known about whether alterations of interhemispheric functional coordination occur in patients with PDM.

We hypothesized that patients with PDM have poor coordination between left and right hemispheric activity compared with healthy individuals, which may be associated with the pathophysiology of PDM. To explore this hypothesis, we gathered rs-fMRI and clinical data from patients with PDM and healthy controls (HCs), and then determined variation in VMHC between the two groups. We also identified seed-based FC changes in brain regions with significant VMHC in patients with PDM. Finally, the correlation of altered VMHC and FC with clinical scores of patients with PDM was investigated.

We present the following article in accordance with the Materials Design Analysis Reporting (MDAR) checklist (available at https://dx.doi.org/10.21037/qims-21-731).

\section{Methods}

\section{Study participants}

All participants gave written informed consent before participating in the study. The study protocol was approved by the Medical Ethics Committee of Beijing University of Chinese Medicine. The study was conducted in adherence with the ethical principles of the Declaration of Helsinki (as revised in 2013).

From December 2016 to November 2018, all patients with PDM from multiple colleges in Beijing were recruited. All potential participants were screened and their eligibility assessed by an experienced doctor. In compliance with the diagnostic criteria of the Clinical Practice Guideline of PDM published in July 2017 (1), to be included in the study participants needed to fulfil the following criteria: (I) female; (II) aged between 16 and 30 years old; (III) right- 
handed; (IV) had not given birth; (V) had regular menstrual periods (occurring every 28 7 days); (VI) had experienced a pain episode lasting for at least six months; (VII) had not received any treatment for PDM in the first month before the study; (VIII) not a long-term caffeine consumer; (IX) had not consumed tea in the three days preceding the MRI scan; and $(\mathrm{X})$ had an average visual analogue score (VAS) for dysmenorrhea equal to or greater than 40 (with $0=$ no pain and $100=$ worst pain imaginable) during the three months before enrollment.

The HCs fulfilled the same criteria, excluding those relating to PDM. The HCs were in good health, lacked dysmenorrhea, and did not show any structural brain abnormality on MRI.

Participants were excluded if they had any of the following: (I) pelvic organ diseases; (II) any other neurological diseases or psychiatric disorders; (III) severe life-threatening diseases; (IV) a history of alcohol or drug abuse; (V) any contraindications for MRI; and (VI) severe structural abnormalities in the brain.

In our study, 40 patients with PDM and $44 \mathrm{HCs}$ met the inclusion criteria and underwent rs-fMRI examination. Of these participants, 5 patients with PDM and 3 HCs were eliminated from the study due to excessive head motion. Ultimately, the data of 35 patients with PDM and 41 HCs were analyzed.

All participants completed VAS pain (VAS-P) and VAS anxiety (VAS-A) questionnaires, which assessed on their levels of pain and related anxiety in the first three menstrual cycles before the study. VAS scores range from 0 (no pain or anxiety) to 100 (worst pain or anxiety imaginable).

\section{MRI data acquisition}

The imaging data were acquired on a 3.0 Tesla scanner (Magnetom Skyra, Siemens, Erlangen, Germany) at the Department of Radiology in Beijing Hospital of Traditional Chinese Medicine, Capital Medical University. For all participants, the MRI scan was performed on days 1 to 3 of the menstrual cycle. The examination was performed with the participant in a supine position on the scanner bed, with sponge cushions on both sides on their ears to minimize head motion. Participants were instructed to close their eyes, stay awake, relax, remain as still as possible, and avoid thinking about anything. After the scan, each participant was asked whether they had fallen asleep during the examination.

T1-weighted images were acquired (repetition time 2,300 ms; echo time $2.32 \mathrm{~ms}$; slice thickness $0.9 \mathrm{~mm}$; slices 192; flip angle $8^{\circ}$; field of view $240 \mathrm{~mm} \times 240 \mathrm{~mm}$; matrix size $256 \times 256$ ). An echo-planar imaging sequence was used for rs-fMRI (repetition time 3,000 ms; echo time $30 \mathrm{~ms}$; voxel size $2.3 \times 2.3 \times 3.0 \mathrm{~mm}^{3}$; distance factor $25 \%$; slices 40 ; flip angle $90^{\circ}$; field of view $220 \mathrm{~mm} \times 220 \mathrm{~mm}$; matrix size 94×94; measurements 250).

\section{Resting-state fMRI preprocessing and calculation}

All rs-fMRI data were preprocessed and calculated using the Data Processing \& Analysis of Brain Imaging (DPABI, http://rfmri.org/DPABI) and Statistical Parametric Mapping 12 (SPM 12) software in MATLAB R2015b (http://www.fil. ion.ucl.ac.uk/spm).

The volumes from the first 10 seconds were discarded to ensure the signal equilibrium and allow for participants to adapt to scanning. The remaining 240 functional images were corrected using slice timing to adjust for differences in slice acquisition times. Afterward, head motion correction was performed, and volumes with excessive motion (displacement $>3 \mathrm{~mm}$ or 3 degrees in any direction) were removed. The remaining images were co-registered to T1 images for spatial normalization based on the Montreal Neurological Institute protocol and resampled at an isotropic voxel size of $3 \mathrm{~mm}^{3}$. The nuisance covariates, which included white matter signal, cerebral spinal fluid signal, and Friston 24 head motion parameters, were regressed. The time series of each participant was then temporally bandpass filtered $(0.01-0.1 \mathrm{~Hz})$ to reduce low-frequency drift and highfrequency noise. Finally, linear trends were removed, and images were smoothed with a three-dimensional Gaussian kernel of $4 \mathrm{~mm}$ full width at half maximum.

The standardized time series of each voxel was extracted to calculate the Pearson correlation coefficient (namely, the VMHC value) between bilateral symmetrical regions for each participant. These correlation coefficients were then converted to Z-values using Fisher Z-transformation, and the results were further analyzed between groups.

Brain areas showing significant group differences in VMHC maps were selected as seed regions to investigate FC changes. Correlation analysis was conducted between the averaged time series of each seed and the time series of the remaining voxels of the participant's whole brain. To improve the normality of correlation coefficients, Fisher's r-to-z transformation was used.

\section{Statistical analysis}

SPSS 22.0 (IBM Corporation, Armonk, NY, USA) was used 
Table 1 Baseline characteristics of patients with PDM and HCs

\begin{tabular}{lccc}
\hline Parameter & PDM group $(\mathrm{n}=35)$ & $\mathrm{HCs}(\mathrm{n}=41)$ & $\mathrm{P}$ \\
\hline Age (year) & $23.9 \pm 2.6$ & $24.9 \pm 1.9$ & 0.252 \\
Menstrual cycle (day) & $29.9 \pm 3.3$ & $30.5 \pm 4.1$ & 0.661 \\
Menstrual phase (day) & $4-7$ & $4-7$ & 0.962 \\
Onset age of & $16.8 \pm 2.8$ & - & - \\
dysmenorrhea (year) & & & - \\
VAS-A & $57.2 \pm 27.4$ & - & - \\
VAS-P & $71.6 \pm 18.0$ & - & - \\
\hline
\end{tabular}

Data are mean \pm standard deviation; the menstrual phase is maximum and minimum values. PDM, primary dysmenorrhea; HCs, healthy controls; VAS-A, visual analogue score for anxiety; VAS-P, visual analogue score for pain.

for all clinical data analyses. A two-sample $t$-test was used to analyze differences in clinical data between the two groups including age, menstrual cycle, and phase. The statistical significance threshold was set at $\mathrm{P}<0.05$.

Group comparisons of global VMHC and seed-based FC between the patients with PDM and HCs were conducted using a two-sample $t$-test in DPABI. Significance was set at a Gaussian random field theory-corrected voxel level of $\mathrm{P}<0.001$ and a cluster level $<0.05$ for multiple comparisons.

Pearson correlation analysis was also conducted between abnormal VMHC and FC and dysmenorrhea onset age, symptom duration, and participant VAS-A and VAS-P scores, with the significance level set at $\mathrm{P}<0.05$.

\section{Results}

\section{Baseline demographic and clinical characteristics}

The final analysis included 35 patients with PDM and 41 HCs. There were no significant statistical differences between the two groups in age, menstrual cycle, or phase $(\mathrm{P}>0.05)$ (Table 1).

\section{VMHC differences between the groups}

Compared with the HCs, patients with PDM showed enhanced VMHC in the bilateral orbital part of the superior frontal gyrus and bilateral middle frontal gyrus (Figure 1, Table 2). The HCs showed no decreased VMHC in any brain region.

\section{Seed-based FC differences between the two groups}

As shown in Figure 2 and Table 3, seed-based FC analysis showed that patients with PDM had increased FC of the left orbital part of the superior frontal gyrus with the right caudate nucleus, the right inferior occipital gyrus, and the left postcentral gyrus. The FC of the right orbital part of the superior frontal gyrus with the left rectus gyrus, the left caudate nucleus, and the left orbital part of the medial frontal gyrus was increased. The FC of the left middle frontal gyrus with the right anterior cingulate gyrus and the left superior parietal gyrus was also increased, as was the FC of the right middle frontal gyrus with the left anterior cingulate gyrus. No decreases in FC were observed in brain regions of patients with PDM compared with the HCs.

\section{Correlations between seed-based FC and clinical parameters}

In patients with $\mathrm{PDM}$, increased $\mathrm{FC}$ between the left middle frontal gyrus and the right anterior cingulate gyrus was negatively correlated with an increase in the reported VAS-P score $(\mathrm{r}=-0.341 ; \mathrm{P}=0.045)$. Results are shown in Figure 3.

\section{Discussion}

The main finding of our study is that VMHC was dramatically increased in the bilateral orbital part of the superior frontal gyrus and the bilateral middle frontal gyrus in patients with PDM and ongoing menstrual pain compared with HCs. Further seed-based FC analysis indicated that patients with PDM displayed enhanced connectivity between the aforementioned areas and some pain-related brain structures, such as the caudate nucleus, the postcentral gyrus, and the anterior cingulate gyrus. Hyperconnectivity of the left middle frontal gyrus with the right anterior cingulate gyrus was negatively correlated with pain severity. Disrupted interhemispheric FC was found in pain-related brain regions, attention networks, and reward circuits involved in multiple dimensions of pain modulation, and these anomalies may be the central mechanism underlying pain related to PDM.

\section{Increased FC in the sensory-discriminative aspect of pain}

Pain is a widely recognized but complicated experience, 

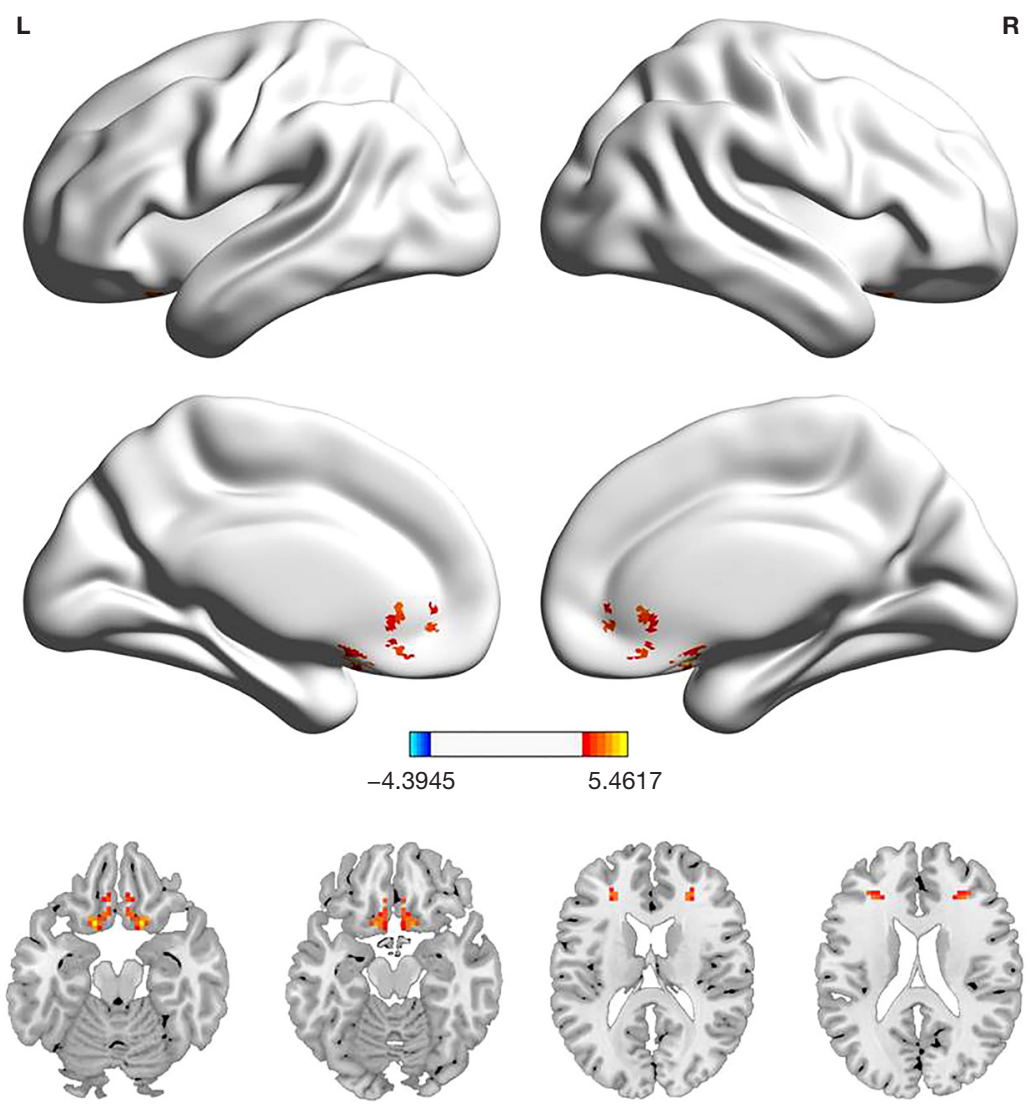

$-19 \mathrm{~mm}$

$-16 \mathrm{~mm}$

$17 \mathrm{~mm}$

$20 \mathrm{~mm}$

Figure 1 Brain areas showing increased VMHC in patients with PDM compared with HCs. Warm colors indicate increased VMHC values. Gaussian Random Field correction was used to test for statistical significance (voxel $\mathrm{P}<0.001$; cluster $\mathrm{P}<0.05$; two-tailed). VMHC, voxelmirrored homotopic connectivity; PDM, primary dysmenorrhea; HCs, healthy controls.

Table 2 Brain regions showing VMHC changes between patients with PDM and HCs

\begin{tabular}{lccc}
\hline Regions (aal) & Brain region & Cluster size & Coordinates in MNI (x, y, z) \\
\hline Frontal_Sup_Orb_L & Left orbital part of superior frontal gyrus & 2,835 & $-15,15,-18$ \\
Frontal_Sup_Orb_R & Right orbital part of superior frontal gyrus & 2,835 & 5.46 \\
Frontal_Mid_L & Left middle frontal gyrus & 513 & 5.46 \\
Frontal_Mid_R & Right middle frontal gyrus & 513 & $-30,33,24$ \\
\hline
\end{tabular}

VMHC, voxel-mirrored homotopic connectivity; PDM, primary dysmenorrhea; HCs, healthy controls; MNI, Montreal Neurological Institute.

and the processing of pain is considered to be fundamentally encoded by a sensory-discriminative aspect. Our study found hyperconnectivity of the orbital part of the superior frontal gyrus (which belongs to the orbitofrontal cortex, OFC) with the caudate nucleus, the postcentral gyrus, and other brain regions in patients with PDM. The caudate nucleus has crucial functional interactions with the high-level cortex which receives input from the medial OFC involved in the cognitive aspect of pain processing (20). Previous morphological surveys of grey matter changes found that the subcortical volume of the caudate nucleus was decreased, which might cause unpleasant or painful visceral sensations for patients with PDM (21). A recent study also supported the idea that gray matter volume changes in the caudate nucleus are correlated with menstrual pain (22). Functional abnormalities in the caudate nucleus related to pain perception and modulation have been observed in other 


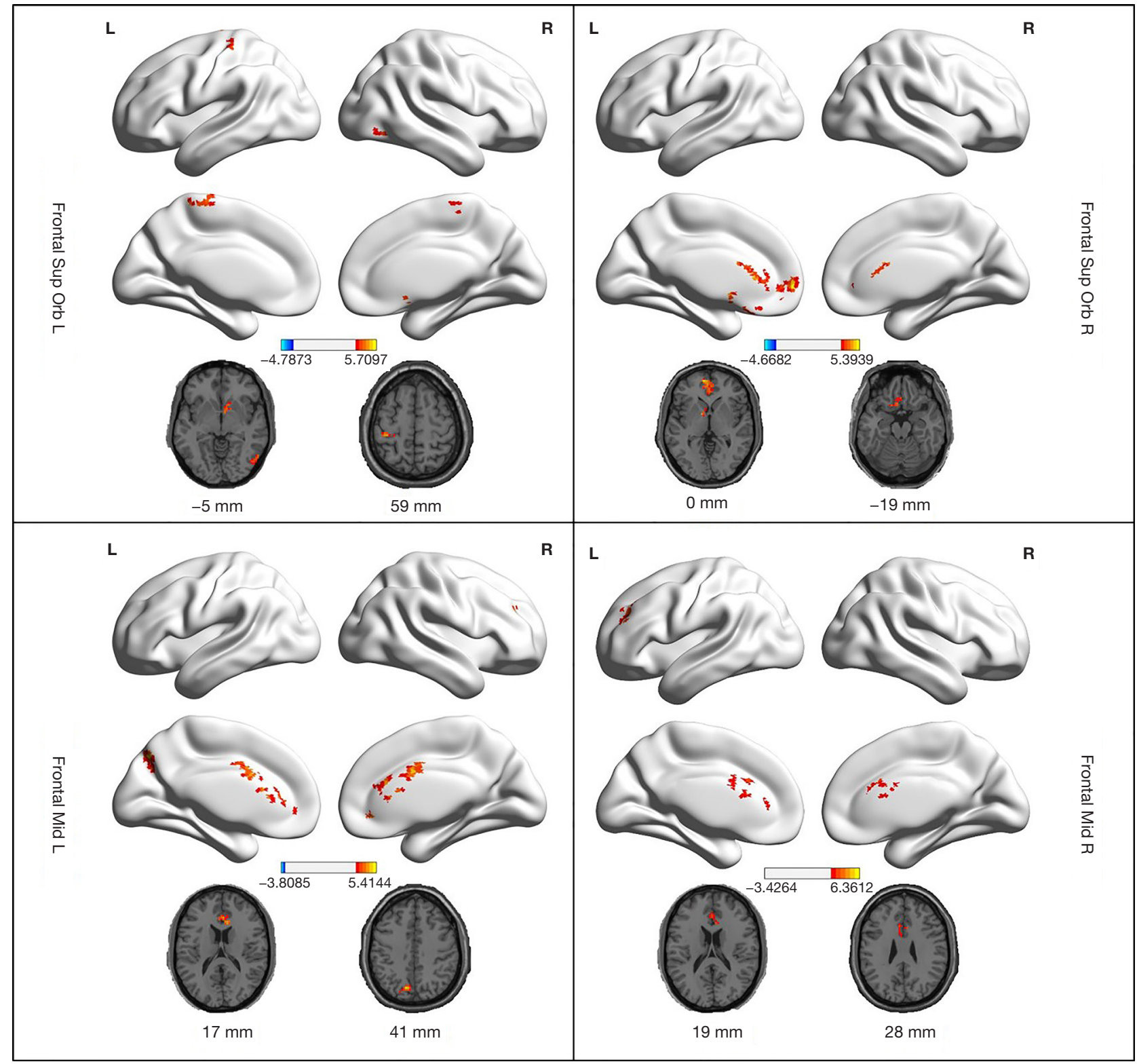

Figure 2 Differences in seed-based FC between patients with PDM and HCs. Warm colors represent increased FC values. Gaussian Random Field correction was used to test for statistical significance (voxel $\mathrm{P}<0.001$; cluster $\mathrm{P}<0.05$; two-tailed). FC, functional connectivity; PDM, primary dysmenorrhea; HCs, healthy controls.

conditions, such as in thermal stimuli and migraine without aura $(23,24)$. Furthermore, the postcentral gyrus belongs to the primary somatosensory cortex, which is responsible for sensory-discriminative aspects of pain, such as location, intensity, and quality (25). A previous report found that patients with PDM exhibited increased connectivity between the caudal anterior cingulate cortex and the primary somatosensory cortex (26), and another study reported increased regional cortical thickness in this area (21). Our study observed a greater correlation in the FC of cerebral blood flow between the right rolandic operculum and the bilateral postcentral gyrus (27). In summary, disruption of the FC of the OFC with the caudate nucleus and the postcentral gyrus likely contributes to pathologic alterations in the sensorydiscriminative processing of ongoing menstrual pain. 
Table 3 Brain regions with increased seed-based FC in patients with PDM compared with HCs

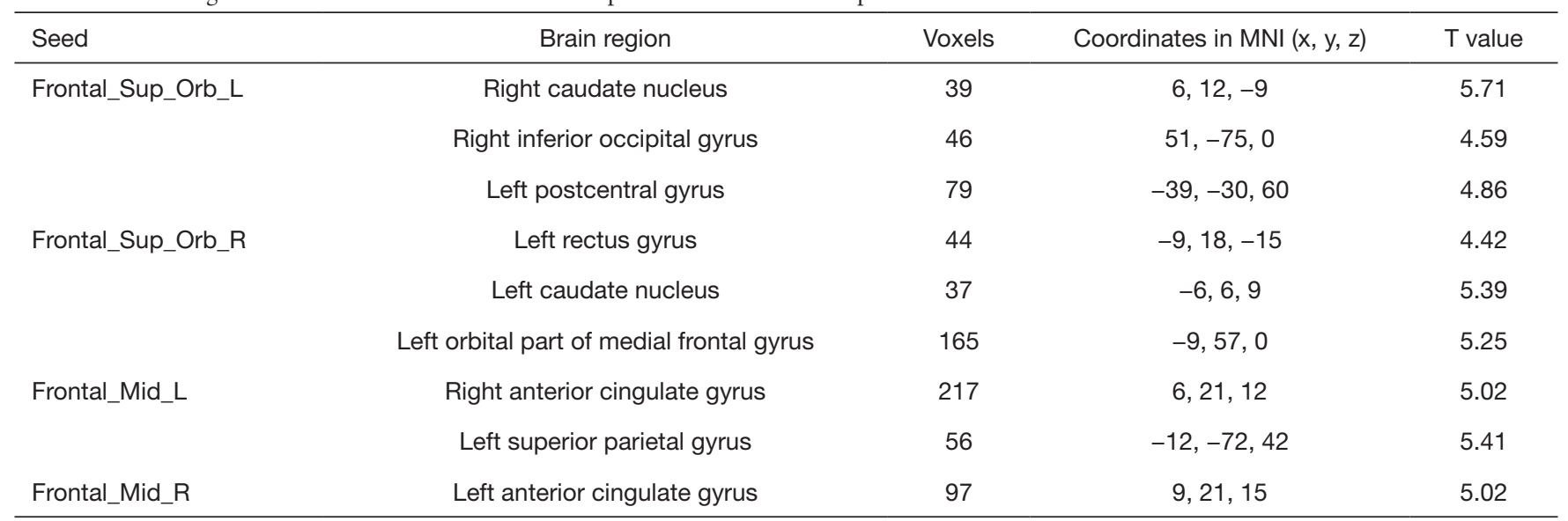

FC, functional connectivity; PDM, primary dysmenorrhea; HCs, healthy controls; Frontal_Sup_Orb_L, left orbital part of the superior frontal gyrus; Frontal_Sup_Orb_R, right orbital part of the superior frontal gyrus; Frontal_Mid_L, left middle frontal gyrus; Frontal_Mid_R, right middle frontal gyrus; MNI, Montreal Neurological Institute.

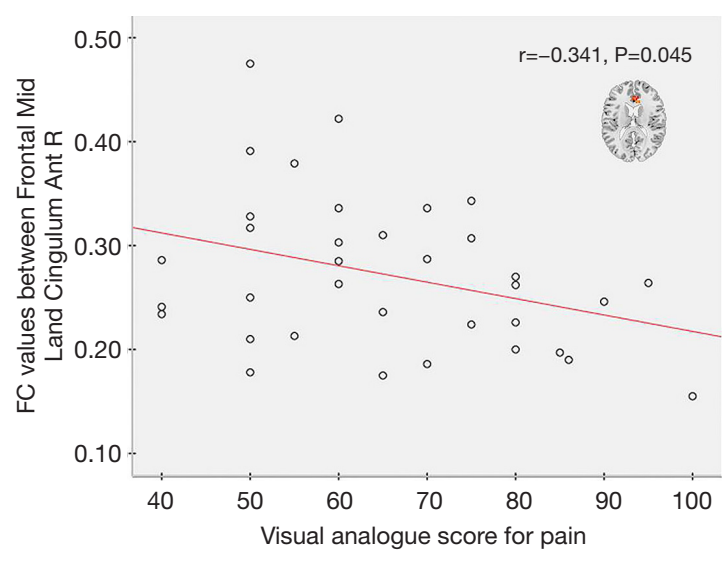

Figure 3 Correlation of increased FC values of the left middle frontal gyrus and the right anterior cingulate gyrus with VAS-P. FC, functional connectivity; VAS-P, visual analogue score for pain.

\section{Increased VMHC and FC in the cognitive-evaluative dimension of pain}

Pain processing is complex and can be influenced by the attention levels of the individual experiencing it, as shown during cognitive modulation of pain. The attention network plays a pivotal role in the regulation of pain-related behavior. The dorsal attention system is involved in topdown attention orientation, while the ventral attention system is associated with attention reorientation in response to salient sensory stimuli (28). The two networks interact during pain processing, and the middle frontal gyrus has been proposed as a region that links them (29). Our study's finding that VMHC was increased in this area might be explained by enhanced interhemispheric coordination between the bilateral middle frontal gyrus, which might strengthen the capacity of an individual's cognitiveevaluative modulation of unpleasant menstrual pain. In agreement with this, reports have shown that the middle frontal gyrus is related to the cognitive evaluation and modulation of pain in post-traumatic headache (30) and chronic lower back-related leg pain (19).

Furthermore, we also discovered increased FC of the middle frontal gyrus with the anterior cingulate gyrus. Substantial evidence shows that the anterior cingulate gyrus is implicated in emotional, attentional, sensory, and pain processing $(31,32)$. Neuroimaging studies have shown that patients with PDM have increased low-frequency fluctuation in the anterior cingulate gyrus and decreased FC with the precuneus. The authors of this previous work speculated that these findings reflect disturbances in the attentional and emotional modulation of pain in patients with PDM (11). Other studies have found that abnormal $\mathrm{FC}$ of the anterior cingulate gyrus might be involved in PDM-related dysfunction of pain perception, regulation, and affect (26). Our findings reveal a negative correlation between pain intensity and FC of the left middle frontal gyrus with the right cingulate gyrus. This finding suggests that an individual's pain is ameliorated when the attention network is active. The hyperconnectivity between these regions may be a beneficial adaptation for the patient. 
Although this finding is interesting, it should be cautiously interpreted, given that the correlation is weak and does not indicate a causal relationship between attention network functionality and pain severity. Further clarification through longitudinal intervention studies is needed.

\section{Increased VMHC in the motivational-affective aspect of pain}

Experiencing a painful stimulus is a motivationally prominent event, while pain-relief represents a potential reward and may promote a positive emotional state. Brain reward circuits (33) play a crucial role in reward processing, motivation, and pain modulation. This circuitry encompasses regions including the nucleus accumbens, the prefrontal cortex, the ventral tegmental area, and the lateral OFC. The OFC has been implicated in sensory integration, emotion, reward-related behaviors, and pain modulation $(34,35)$. Most of what is known about PDM modulationassociated reward circuits has come from recent rs-fMRI studies. One study (14) investigated the FC changes of the reward system during the pain-free periovulatory phase in patients with PDM compared with HCs. This study revealed reduced connectivity between the right nucleus accumbens and the right OFC. Furthermore, the decreased $\mathrm{FC}$ of the right nucleus accumbens-ventral tegmental area had a negative relationship with the level of prostaglandins, suggesting that pain relief in the menstrual phase was accompanied by weakening in the FC of the reward system during the ovulatory period. Consistent with this study, we also observed decreased FC between the left amygdala with the bilateral OFC, and the right amygdala with the right OFC, in patients with PDM in a pain-free state (13). These findings provide scientific evidence to support the contention that a dysfunctional reward network might be involved in the pathophysiology of PDM.

In contrast to previous studies, our study assessed FC changes in the reward system during the menstrual period. FC was found to be enhanced in the bilateral OFC, and hyperconnectivity of the OFC with other pain-associated regions. Our findings suggest that as emotional regulation of reward and motivation can be used by patients who are seeking to alleviate their menstrual cramps. We speculate that activation of the reward system is an active response to pain in patients with dysmenorrhea. In any case, the reward network appears to be associated with a central mechanism underlying PDM. In support of our observations, a previous study using fluorodeoxyglucose positron emission tomography showed that patients with PDM had greater metabolism in the OFC during their menstrual phase than did HCs (36). We believe that activation of the thalamoorbitofrontal-prefrontal networks may participate in pain onset and hyperalgesia in PDM. In addition to functional and metabolic abnormalities, patients with PDM were also reported to have increased cortical thickness in the OFC, which was positively associated with pain duration (21).

\section{Limitations}

Although our findings are promising, several limitations should be noted. First, increased VMHC was observed in patients with PDM during pain, and whether homotopic connectivity is abnormal in a pain-free state is still unclear. Research is needed in future to investigate the menstrual and ovulatory phases, including different menstrual cycles, to observe interhemispheric functional coordination. Second, our study only compared patients with PDM without recent treatment and HCs. A longitudinal study with an intervention is essential to support our understanding of possible causal links between VMHC and PDM. Third, our study did not have a large sample size, which may have affected the reliability and stability of the neuroimaging results. Therefore, more participants will need to be recruited to increase the statistical power in future studies.

\section{Conclusions}

Our study has demonstrated that ongoing menstrual pain is accompanied by increased VMHC in the bilateral orbital part of the superior frontal gyrus and the bilateral middle frontal gyrus. Our study also discovered enhanced FC between these regions and brain regions involved in pain processing in patients with PDM as compared to HCs. Our results provide evidence of interhemispheric dysfunction related to PDM and support the finding that activation from pain-related regions to attention networks and within the reward system may be involved in different aspects of the experience of dysmenorrhea.

\section{Acknowledgments}

Funding: This work was supported by the National Natural Science Foundation of China (81503652), University-Level Longitudinal Research Development Foundation of Beijing University of Chinese Medicine (2020-ZXFZJJ-035), and Beijing Municipal Administration of Hospitals Incubating 
Program (PZ2021016).

\section{Footnote}

Reporting Checklist: The authors have completed the MDAR checklist. Available at https://dx.doi.org/10.21037/ qims-21-731

Conflicts of Interest: All authors have completed the ICMJE uniform disclosure form (available at https://dx.doi. org/10.21037/qims-21-731). The authors have no conflicts of interest to declare.

Ethical Statement: The authors are accountable for all aspects of the work in ensuring that questions related to the accuracy or integrity of any part of the work are appropriately investigated and resolved. All subjects gave written informed consent prior to participation in the study. The study protocol was approved by the Medical Ethics Committee of Beijing University of Chinese Medicine. The study was conducted in conformity with the ethical principles of Declaration of Helsinki (as revised in 2013).

Open Access Statement: This is an Open Access article distributed in accordance with the Creative Commons Attribution-NonCommercial-NoDerivs 4.0 International License (CC BY-NC-ND 4.0), which permits the noncommercial replication and distribution of the article with the strict proviso that no changes or edits are made and the original work is properly cited (including links to both the formal publication through the relevant DOI and the license). See: https://creativecommons.org/licenses/by-nc-nd/4.0/.

\section{References}

1. Burnett M, Lemyre M. No. 345-Primary Dysmenorrhea Consensus Guideline. J Obstet Gynaecol Can 2017;39:585-95.

2. Ferries-Rowe E, Corey E, Archer JS. Primary Dysmenorrhea: Diagnosis and Therapy. Obstet Gynecol 2020;136:1047-58.

3. Abu Helwa HA, Mitaeb AA, Al-Hamshri S, Sweileh WM. Prevalence of dysmenorrhea and predictors of its pain intensity among Palestinian female university students. BMC Womens Health 2018;18:18.

4. Ameade EPK, Amalba A, Mohammed BS. Prevalence of dysmenorrhea among University students in Northern Ghana; its impact and management strategies. BMC Womens Health 2018;18:39.
5. Yesuf TA, Eshete NA, Sisay EA. Dysmenorrhea among University Health Science Students, Northern Ethiopia: Impact and Associated Factors. Int J Reprod Med 2018;2018:9730328.

6. Hu Z, Tang L, Chen L, Kaminga AC, Xu H. Prevalence and Risk Factors Associated with Primary Dysmenorrhea among Chinese Female University Students: A Crosssectional Study. J Pediatr Adolesc Gynecol 2020;33:15-22.

7. Dun WH, Yang J, Yang L, Ding D, Ma XY, Liang FL, von Deneen KM, Ma SH, Xu XL, Liu J, Zhang M. Abnormal structure and functional connectivity of the anterior insula at pain-free periovulation is associated with perceived pain during menstruation. Brain Imaging Behav 2017;11:1787-95.

8. Yang L, Dun W, Li K, Yang J, Wang K, Liu H, Liu J, Zhang $\mathrm{M}$. Altered amygdalar volume and functional connectivity in primary dysmenorrhoea during the menstrual cycle. Eur J Pain 2019;23:994-1005.

9. Quan S, Yang J, Dun W, Wang K, Liu H, Liu J. Prediction of pain intensity with uterine morphological features and brain microstructural and functional properties in women with primary dysmenorrhea. Brain Imaging Behav 2021;15:1580-8.

10. Wu TH, Tu CH, Chao HT, Li WC, Low I, Chuang CY, Yeh TC, Cheng CM, Chou CC, Chen LF, Hsieh JC. Dynamic Changes of Functional Pain Connectome in Women with Primary Dysmenorrhea. Sci Rep 2016;6:24543.

11. Liu P, Liu Y, Wang G, Yang X, Jin L, Sun J, Qin W. Aberrant default mode network in patients with primary dysmenorrhea: a fMRI study. Brain Imaging Behav 2017;11:1479-85.

12. Han F, Liu H, Wang K, Yang J, Yang L, Liu J, Zhang M, Dun W. Correlation Between Thalamus-Related Functional Connectivity and Serum BDNF Levels During the Periovulatory Phase of Primary Dysmenorrhea. Front Hum Neurosci 2019;13:333.

13. Shen Z, Yu S, Wang M, She T, Yang Y, Wang Y, Wei W, Guo X, Hu Y, Liang F, Yang J. Abnormal amygdala resting-state functional connectivity in primary dysmenorrhea. Neuroreport 2019;30:363-8.

14. Zhang Q, Yu S, Wang Y, Wang M, Yang Y, Wei W, Guo X, Zeng F, Liang F, Yang J. Abnormal reward system network in primary dysmenorrhea. Mol Pain 2019;15:1744806919862096.

15. Zuo XN, Kelly C, Di Martino A, Mennes M, Margulies DS, Bangaru S, Grzadzinski R, Evans AC, Zang YF, Castellanos FX, Milham MP. Growing together and growing apart: regional and sex differences in the lifespan 
developmental trajectories of functional homotopy. J Neurosci 2010;30:15034-43.

16. Stark DE, Margulies DS, Shehzad ZE, Reiss P, Kelly AM, Uddin LQ, Gee DG, Roy AK, Banich MT, Castellanos FX, Milham MP. Regional variation in interhemispheric coordination of intrinsic hemodynamic fluctuations. J Neurosci 2008;28:13754-64.

17. Jiang J, Gu L, Bao D, Hong S, He W, Tan Y, Zeng X, Gong H, Zhang D, Zhou F. Altered homotopic connectivity in postherpetic neuralgia: a resting state fMRI study. J Pain Res 2016;9:877-86.

18. Dong ZZ, Zhu FY, Shi WQ, Shu YQ, Chen LL, Yuan Q, Lin Q, Zhu PW, Liu KC, Min YL, Ye L, Shao Y. Abnormalities of interhemispheric functional connectivity in individuals with acute eye pain: a resting-state fMRI study. Int J Ophthalmol 2019;12:634-9.

19. Zhang Y, Zhu Y, Pei Y, Zhao Y, Zhou F, Huang M, Wu L, Zhang D, Gong H. Disrupted interhemispheric functional coordination in patients with chronic low back-related leg pain: a multiscale frequencyrelated homotopic connectivity study. J Pain Res 2019;12:2615-26.

20. Kringelbach ML, Rolls ET. The functional neuroanatomy of the human orbitofrontal cortex: evidence from neuroimaging and neuropsychology. Prog Neurobiol 2004;72:341-72.

21. Liu P, Yang J, Wang G, Liu Y, Liu X, Jin L, Liang F, Qin W, Calhoun VD. Altered regional cortical thickness and subcortical volume in women with primary dysmenorrhoea. Eur J Pain 2016;20:512-20.

22. Tu CH, Niddam DM, Yeh TC, Lirng JF, Cheng CM, Chou CC, Chao HT, Hsieh JC. Menstrual pain is associated with rapid structural alterations in the brain. Pain 2013;154:1718-24.

23. Freund W, Klug R, Weber F, Stuber G, Schmitz B, Wunderlich AP. Perception and suppression of thermally induced pain: a fMRI study. Somatosens Mot Res 2009;26:1-10.

24. Gao Q, Xu F, Jiang C, Chen Z, Chen H, Liao H, Zhao L. Decreased functional connectivity density in pain-related brain regions of female migraine patients without aura. Brain Res 2016;1632:73-81.

25. Kenshalo DR Jr, Chudler EH, Anton F, Dubner R. SI nociceptive neurons participate in the encoding process by which monkeys perceive the intensity of noxious thermal stimulation. Brain Res 1988;454:378-82.

26. Liu P, Liu Y, Wang G, Li R, Wei Y, Fan Y, Yu Y, Deng D, Qin W. Changes of functional connectivity of the anterior cingulate cortex in women with primary dysmenorrhea. Brain Imaging Behav 2018;12:710-7.

27. Zhang YN, Huang YR, Liu JL, Zhang FQ, Zhang BY, Wu JC, Ma Y, Xia J, Hao Y, Huo JW. Aberrant restingstate cerebral blood flow and its connectivity in primary dysmenorrhea on arterial spin labeling MRI. Magn Reson Imaging 2020;73:84-90.

28. Legrain V, Damme SV, Eccleston C, Davis KD, Seminowicz DA, Crombez G. A neurocognitive model of attention to pain: behavioral and neuroimaging evidence. Pain 2009;144:230-2.

29. Vossel S, Geng JJ, Fink GR. Dorsal and ventral attention systems: distinct neural circuits but collaborative roles. Neuroscientist 2014;20:150-9.

30. Lu L, Li F, Wang P, Chen H, Chen YC, Yin X. Altered hypothalamic functional connectivity in post-traumatic headache after mild traumatic brain injury. J Headache Pain 2020;21:93.

31. Bantick SJ, Wise RG, Ploghaus A, Clare S, Smith SM, Tracey I. Imaging how attention modulates pain in humans using functional MRI. Brain 2002;125:310-9.

32. Van Oudenhove L, Demyttenaere K, Tack J, Aziz Q. Central nervous system involvement in functional gastrointestinal disorders. Best Pract Res Clin Gastroenterol 2004;18:663-80.

33. Morales M, Margolis EB. Ventral tegmental area: cellular heterogeneity, connectivity and behaviour. Nat Rev Neurosci 2017;18:73-85.

34. Kringelbach ML. The human orbitofrontal cortex: linking reward to hedonic experience. Nat Rev Neurosci 2005;6:691-702.

35. Fumal A, Laureys S, Di Clemente L, Boly M, Bohotin V, Vandenheede M, Coppola G, Salmon E, Kupers R, Schoenen J. Orbitofrontal cortex involvement in chronic analgesic-overuse headache evolving from episodic migraine. Brain 2006;129:543-50.

36. Tu CH, Niddam DM, Chao HT, Liu RS, Hwang RJ, Yeh TC, Hsieh JC. Abnormal cerebral metabolism during menstrual pain in primary dysmenorrhea. Neuroimage 2009;47:28-35.

Cite this article as: Zhang $\mathrm{Y}$, Huang $\mathrm{Y}$, Liu N, Wang Z, Wu J, Li W, Xia J, Liu Z, Li Y, Hao Y, Huo J. Abnormal interhemispheric functional connectivity in patients with primary dysmenorrhea: a resting-state functional MRI study. Quant Imaging Med Surg 2022;12(3):1958-1967. doi: 10.21037/ qims-21-731 